$01 ; 11$

\title{
Моделирование резонансов диэлектрических сфер в терагерцевом диапазоне
}

\author{
() Д.В. Стороженко ${ }^{1}$, В.П. Дзюба ${ }^{1}$, Ю.Н. Кульчин ${ }^{1,2}$ \\ ${ }^{1}$ Институт автоматики и процессов управления ДВО РАН, \\ Владивосток, Россия \\ ${ }^{2}$ Дальневосточный федеральный университет, \\ Владивосток, Россия \\ E-mail: dbrados@dvo.ru
}

Поступило в Редакцию 21 ноября 2017 г.

Исследованы резонансные свойства диэлектрических сфер субволновых размеров в оптическом и терагерцевом диапазонах. Методом конечных элементов смоделировано распределение вектора напряженности электрического поля в сфере, облученной плоской поляризованной волной. Наглядно показана принципиальная возможность использования резонансов различных порядков, возникающих в диэлектрических сферах, для усиления тока в проводящей антенне с размером, в несколько раз меньшим длины волны. В случаях резонансов на коротких участках спектра возможно усиление плотности тока до 25-30 dB по сравнению со случаем отсутствия диэлектрической сферы вокруг антенны.

DOI: $10.21883 /$ PJTF.2018.16.46479.17127

Освоение терагерцевого $(\mathrm{THz})$ или субмиллиметрового диапазона $(0.1-10 \mathrm{THz}$ или $30-3000 \mu \mathrm{m})$ электромагнитного излучения в наши дни остается актуальным. Новые приборы, применяющие эту часть спектра, способны на качественно новом уровне расширить возможности человечества. Однако, несмотря на то что перспективы THz-диапазона известны давно, его массовое применение все еще остается ограниченным из-за физических особенностей поведения материалов, а также проблем, связанных с созданием источников и детекторов излучения. Настоящая работа касается проблемы детектирования излучения. Большинство устройств, реализованных по типу болометров на горячих электронах (HEB) или однофотонных транзисторов (SFT), обладает 
низкой шумностью $\left(\mathrm{NEP}=10^{-19} \mathrm{~W} / \mathrm{Hz}^{0.5}\right.$ для устройств типа HEB, $\mathrm{NEP}=10^{-20} \mathrm{~W} / \mathrm{Hz}^{0.5}$ для SFT), однако для их работы необходима низкая (порядка единиц градусов Кельвина) температура [1-4]. Детекторы на основе джозефсоновских переходов в высокотемпературных сверхпроводниках работают уже в диапазоне до $80 \mathrm{~K}$ и обладают лучшим быстродействием (до нескольких $\mathrm{MHz}$ ), а уровень их шумности составляет $\mathrm{NEP}=10^{-13}-10^{-15} \mathrm{~W} / \mathrm{Hz}^{0.5}[5]$. Тем не менее такая рабочая температура ограничивает применение детекторов лишь использованием в составе космических телескопов или лабораторных установок. Среди детекторов, работающих при комнатной температуре, устройства на основе полупроводников с барьером Шоттки имеют наилучший уровень шумности $\mathrm{NEP}=10^{-10} \mathrm{~W} / \mathrm{Hz}^{0.5}$, при этом их быстродействие может составить до $20 \mathrm{GHz}$ [6]. Увеличение соотношения сигнал/шум таких детекторов крайне актуально, поскольку необходимо увеличивать дальность обнаружения слабых сигналов сквозь слой поглощающей атмосферы. Для приема сигнала в таких устройствах применяется встроенная антенна из проводящего материала различной формы, например штыревая, спиральная и патч-антенна [7]. Размер антенны, как правило, выбирается равным половине длины волны излучения для работы в основном резонансе. При этом антенны перечисленных типов имеют коэффициент усиления не более $7-10 \mathrm{dBi}$ в горизонтальной плоскости. Основная цель настоящей работы - исследование возможности усиления сигнала THz-диапазона в проводящей антенне путем включения ее в объемную диэлектрическую сферу, резонансные свойства которой зависят от размеров и показателя преломления вещества. Из теории рассеяния Ми известно, что диэлектрические частицы сферической формы с близкими к длине волны размерами имеют резонансные свойства. В недавних работах показаны резонансные свойства рассеяния и пропускания сферических частиц полупроводников и диэлектриков в оптическом диапазоне и экспериментально продемонстрирована направленная диэлектрическая антенна в микроволновом диапазоне $[8,9]$. При этом внутренняя структура распределения напряженности электрического поля в случаях высших порядков в этих работах не рассматривалась. Для оценки резонансных свойств таких антенн в оптическом и THz-диапазонах мы провели моделирование численным методом конечных элементов (FEM) в частотной области. 


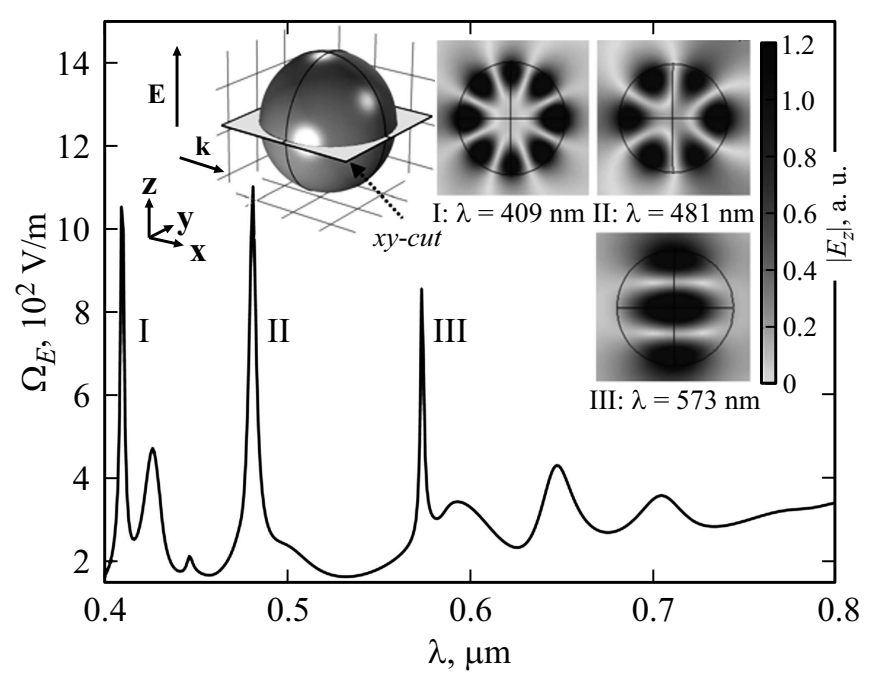

Рис. 1. Результат расчета $\Omega_{E}$ для оптического диапазона. На вставках геометрия модели с $\varnothing=0.31 \mu \mathrm{m}$ (слева) и распределение $\left|E_{z}\right|$ в $x y$-сечении сферы в резонансных случаях (справа).

Расчетная модель представляет собой результат решения уравнения Максвелла методом конечных элементов в частотной области при заданной геометрии

$$
\nabla \times \mu^{-1}(\nabla \times \mathbf{E})-k_{0}^{2}\left(\varepsilon_{r}-\frac{j \sigma}{\omega \varepsilon_{0}}\right) \mathbf{E} .
$$

Здесь $\varepsilon_{r}=(n-i k)^{2}, \quad \sigma=0$ для диэлектрического материала и $\sigma=5.4 \cdot 10^{7} \mathrm{~S} / \mathrm{m}$ для проводящего материала, $\mu_{r}=1 \mu_{0}$, где $\mu_{0}-$ магнитная постоянная (в единицах системы СИ). Результат расчета напряженности электрического поля для сферической частицы из $\mathrm{TiO}_{2}$ в оптическом диапазоне $(\lambda=0.4-0.8 \mu \mathrm{m})$ представлен на рис. 1 для наглядной демонстрации резонансных свойств диэлектрических сфер. Сфера окружена воздухом, а излучение представляется линейно поляризованной плоской волной, вектор электрического поля $\mathbf{E}$ ориентирован

Письма в ЖТФ, 2018, том 44, вып. 16 
вдоль оси $z$ с волновым вектором $\mathbf{k}$

$$
E(\mathbf{r})=E_{0} e^{i\left((\mathbf{k}, \mathbf{r})-\omega t+\varphi_{0}\right)},
$$

где $\mathbf{r}$ - радиус-вектор точки пространства, $\omega-$ круговая частота излучения, $\varphi_{0}-$ фаза колебания. Поскольку в результате численного моделирования для каждой точки пространства расчетной модели мы получаем значения напряженности электрического поля, выполняя интегрирование по объему сферы модуля вектора $\left|E_{z}\right|$, по положению максимумов на графике удобно определять длины волн резонансных случаев, в том числе и для высших порядков:

$$
\Omega_{E}(\lambda)=\frac{\oint \oint \oint\left|E_{z}(\lambda)\right|}{V_{s p h}},
$$

где $V_{s p h}$ - объем сферы (в единицах системы СИ), $\Omega_{E}-$ введенная величина средней напряженности электрического поля в объеме сферы. На основе полученного решения волнового уравнения для всего объема модели, показанной на рис. 1, представлено распределение электрического поля $\left|E_{z}\right|$ в $x y$-сечении сферы в случаях резонансов для различных длин волн. Расположение резонансов в спектре зависит от размеров частицы и показателя преломления. Увеличение размера сферы или показателя преломления вещества ведет к смещению резонансных пиков в длинноволновую область. Также в ходе моделирования выявлено, что на добротность резонанса влияет показатель преломления диэлектрика. Для сферы с диаметром $0.31 \mu \mathrm{m}$ дипольный резонанс приходится на красную область спектра $\lambda=0.65 \mu \mathrm{m}$, а так как в этом диапазоне показатель преломления $\mathrm{TiO}_{2}$ меньше, чем в фиолетовой, его амплитуда заметно ниже, чем для резонанса шестого порядка. С уменьшением коэффициента экстинкции $k$ материала увеличивается амплитуда резонанса высших порядков по сравнению с низшими. Анализируя распределение поля в резонансных случаях, можно увидеть, что для высших порядков размер отдельной области концентрации энергии уменьшается. Это можно интерпретировать как изменение диаграммы направленности антенны, которая сужается при увеличении порядка резонанса. Видно, что напряженность вектора $\left|E_{z}\right|$ в резонансных случаях имеет некоторую структуру, которую можно использовать для усиления сигнала в антенне, если расположить ее внутри сферы в одной из точек максимума. Установив характер распределения электрического поля $\left|E_{z}\right|$, мы

Письма в ЖТФ, 2018, том 44, вып. 16 


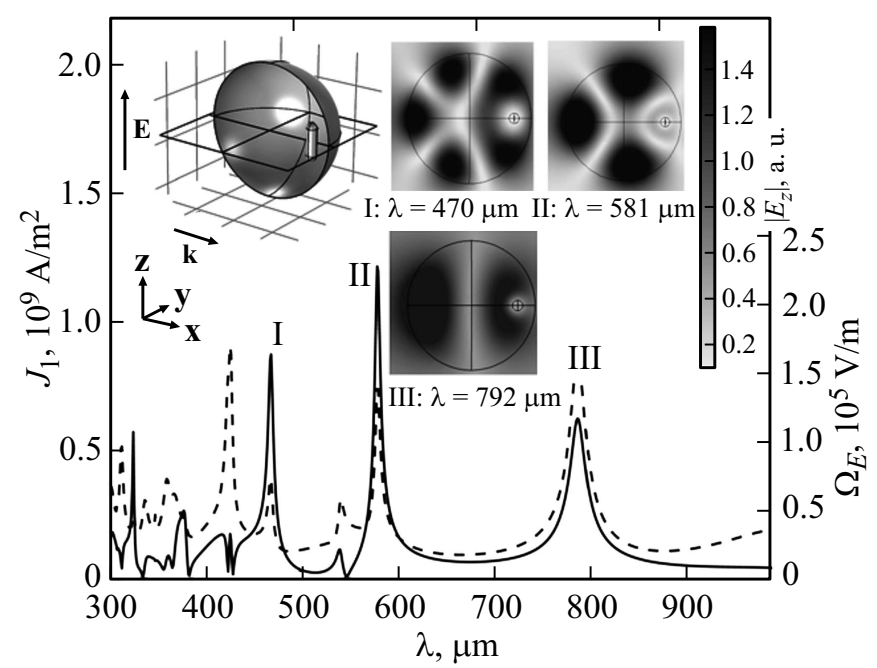

Pис. 2. Спектр средней плотности тока в антенне и средней напряженности поля в сфере. Сплошная линия $-J_{1}(\lambda)$, штриховая $-\Omega_{E}(\lambda)$ при $\varnothing=300 \mu \mathrm{m}$. На вставках показано расположение антенны (слева) и распределение поля $\left|E_{z}\right|$ в $x y$-сечении сферы (справа).

можем определить расположение антенны проводника в сфере (рис. 2) в THz-диапазоне с кратным увеличением геометрических размеров и добавлением проводящей антенны цилиндрической формы, линейный размер которой $(h=120 \mu \mathrm{m})$ меньше диаметра сферы $(\varnothing=300 \mu \mathrm{m})$. При этом цилиндрическая форма проводника из $\mathrm{Cu}$ с проводимостью $\sigma=5.4 \cdot 10^{7} \mathrm{~S} / \mathrm{m}$ содержит геометрические элементы скругления на концах для того, чтобы расчетная область была дифференцируемой. В качестве материала сферы выбран двухкомпонентный диэлектрик $\mathrm{TiO}_{2}-\mathrm{PE}$ (PE - полиэтилен) с объемной концентрацией диоксида титана 0.75. Эффективный показатель преломления смеси составил $n=2.5$ в диапазоне $\lambda=150-600 \mu \mathrm{m}$, а коэффициент $k=1.985 \cdot 10^{-2}$ был пересчитан из измеренного для частоты $1 \mathrm{THz}$ показателя поглощения $\alpha=8.32 \mathrm{~cm}^{-1}$ [10]. На вставках к рис. 2 (справа) показано распределение поля $\left|E_{z}\right|$ в плоскости сечения в резонансных случаях, которое аналогично распределениям поля для оптического диапазона

Письма в ЖТФ, 2018, том 44, вып. 16 


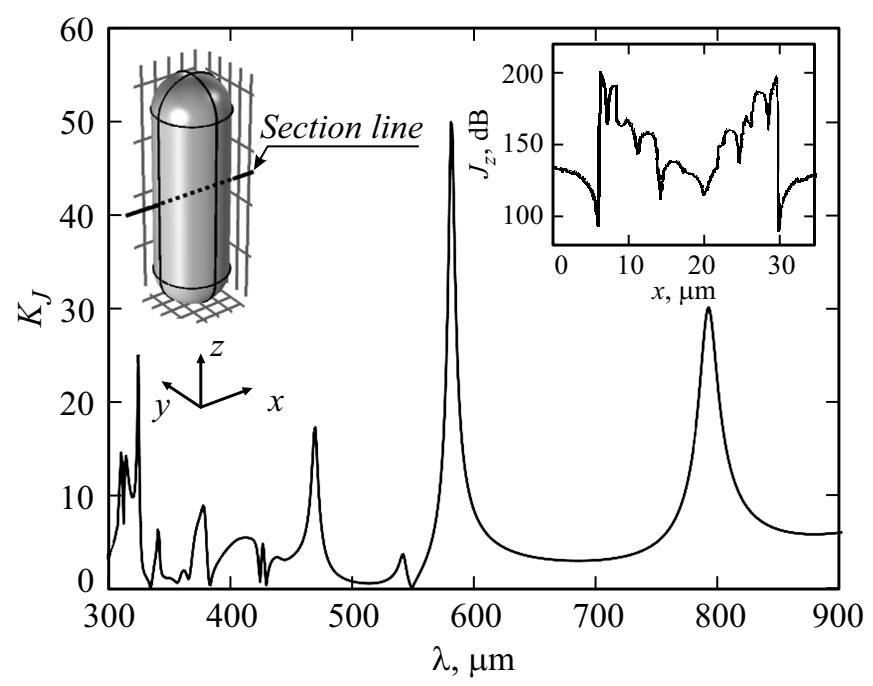

Рис. 3. Коэффициент усиления $K_{J}$. На вставке справа показана плотность тока в логарифмическом масштабе внутри цилиндра по срезу в поперечнике в середине (представлен на вставке слева).

(рис. 1). Различие в положениях длин волн резонансов в спектре, несмотря на кратное соответствие геометрии, обусловлено различием в дисперсии показателя преломления вещества диэлектрика. Согласно измерениям, проведенным в работе [10], в THz-диапазоне показатель преломления можно считать константой, тогда как в оптическом диапазоне для $\mathrm{TiO}_{2}$ наблюдается частотная дисперсия [11]. Рассчитана средняя по объему $V_{c y l}$ цилиндрического проводника, расположенного внутри диэлектрической сферы, плотность тока в направлении $z$ :

$$
J_{1}(\lambda)=\frac{\oint \oint \oint\left|J_{z}(\lambda)\right|}{V_{c y l}} .
$$

Зависимость $J_{1}(\lambda)$ для наглядности отображена в одном масштабе оси $x$ с графиком для $\Omega_{E}$. Как видно из рис. 2 , резонанс плотности тока в основном совпадает с максимумами $\Omega_{E}$. Несовпадение может быть для случаев с неподходящим расположением антенны, поскольку наличие проводника в сфере изменяет распределение поля $E_{z}$ внутри

Письма в ЖТФ, 2018, том 44, вып. 16 
сферы. Увеличение эффективности резонансного усиления возможно за счет оптимальной формы и расположения проводника внутри сферы. При этом нужно отметить, что резонансное распределение поля $E_{z}$ в случаях высших порядков имеет области когерентной фазы, в чем, вероятно, заложена возможность улучшения применения такой конструкции антенного усиления. Для оценки степени усиления сигнала в проводнике цилиндрической формы из меди была рассчитана плотность тока $J_{2}(\lambda)$ в идентичной антенне в случае отсутствия диэлектрической сферы (рис. 3). Коэффициент усиления плотности тока рассчитан по следующему отношению:

$$
K_{J}(\lambda)=J_{1}(\lambda) / J_{2}(\lambda),
$$

где $J_{1}(\lambda)$ - средняя плотность тока в цилиндре внутри диэлектрической сферы, $J_{2}(\lambda)-$ средняя плотность тока в цилиндре в отсутствие диэлектрической сферы. Зависимость $K_{J}(\lambda)$, показанная на рис. 3 , в целом повторяет $J_{1}(\lambda)$. При этом усиление в резонансных случаях достигает 50 раз. Следует отметить, что из-за скин-эффекта в THz-диапазоне разность плотности тока на поверхности и внутри цилиндра может составлять до $100 \mathrm{~dB}$.

Таким образом, в работе продемонстрирована возможность использования резонансов диэлектрических сфер для усиления плотности тока в проводниковой антенне с размером, меньшим, чем длина волны. Размер сфер при этом оказывается сравним с половиной длины волны излучения. Усиление по сравнению со случаем отсутствия диэлектрической сферы в резонансах высоких порядков может достигать $34 \mathrm{~dB}$. Расположение резонансных пиков зависит от размера сфер и показателя преломления диэлектрика. Для увеличения добротности следует использовать диэлектрик с наименьшим коэффициентом экстинкции и наибольшим показателем преломления. Применение такой конструкции позволит на порядок увеличить соотношение сигнал/шум детекторов на основе барьера Шоттки и тем самым приблизить массовое внедрение $\mathrm{THz}$-технологий.

Работа выполнена при частичной поддержке комплексной программы ДВО РАН „Дальний Восток“ (проект № 18-3-023).

6 Письма в ЖТФ, 2018, том 44, вып. 16 


\section{Список литературы}

[1] Komiyama S. // IEEE J. Select. Top. Quant. Electron. 2011. V. 17. N 1. P. 54-66.

[2] Knap W., Rumyantsev S., Vitiello M.S., Coquillat D., Blin S., Dyakonova N., Nagatsuma T. // Nanotechnology. 2013. V. 24. N 21. P. 214002.

[3] Вакс В.Л., Домрачева Е.Г., Ластовкин А.А., Приползин С.И., Собакинская Е.А., Черняева М.Б., Анфертьев В.А. // Вестн. Нижегород. ун-та им. Н.И. Лобачевского. 2013. № 6(1). С. 81-87.

[4] Третьяков И.В., Каурова Н.С., Воронов Б.М., Анфертьев В.А., Ревин Л.С., Вакс В.Л., Гольиман Г.Н. // Письма в ЖТФ. 2016. Т. 42. В. 11. С. 19-26.

[5] Лятти М.В., Ткачев Д.А., Дивин Ю.Я. // Письма в ЖТФ. 2006. Т. 32. В. 19. C. $79-85$.

[6] Wen R., Sun H., Teng T., Li L., Sun X. // J. Semiconductors. 2012. V. 33. N 10. P. 104001.

[7] Liu Z.Y., Liu L.Y., Yang J., Wu N.J. // IEEE Trans. Terahertz Sci. Technol. 2017. V. 7. N 4. P. 455-465. doi: 10.1109/TTHZ.2017.2692040

[8] Krasnok A.E., Filonov D.S., Simovski C.R., Kivshar Y.S., Belov P.A. // Appl. Phys. Lett. 2014. V. 104. N 13. P. 133502. doi:10.1063/1.4869817

[9] Kivshar Y., Miroshnichenko A. // Opt. Photon. News. 2017. V. 28. N 1. P. 24-31.

[10] Ung B., Dupuis A., Stoeffler K., Dubois C., Skorobogatiy M. // J. Opt. Soc. Am. B. 2011. V. 28. N 4. P. 917-921.

[11] Siefke T., Kroker S., Pfeiffer K., Puffky O., Dietrich K., Franta D., Ohlídal I., Szeghalmi A., Kley E.-B., Tünnermann A. // Adv. Opt. Mater. 2016. V. 4. N 11. P. $1780-1786$. 